\title{
Interface Hamiltonians and bulk critical behavior
}

\author{
David A. Huse, Wim van Saarloos, and John D. Weeks \\ AT\&T Bell Laboratories, Murray Hill, New Jersey 07974
}

(Received 18 June 1984)

\begin{abstract}
We examine the derivation and use of a short-ranged $(d-1)$-dimensional interface Hamiltonian to describe properties of a $d$-dimensional liquid-vapor or Ising system near the critical point. We argue that such a simplified description, which ignores bulk excitations ("bubbles" of the opposite phase) and multiple-valued interface configurations ("overhangs") is valid only on length scales larger than the bulk correlation length $\xi_{B}$. Such excitations with wavelengths up to order $\xi_{B}$ are essential for a correct description of the critical fluctuations, and preclude the use of an interface Hamiltonian to study bulk critical properties. This is explicitly demonstrated in $d=2$ by showing that bubbles and overhangs are relevant operators and we argue that this is true in any dimension. (However, these contributions do not necessarily affect the formal perturbation expansion about the degenerate case $d=1$, as carried out by Wallace and Zia.) This viewpoint is implicit in the physical picture Widom used to derive scaling laws relating interface and bulk critical properties. The longwavelength fluctuations accurately described by an interface Hamiltonian produce a "wandering" of the interface, but this plays no important role in the critical behavior and can be reconciled with Widom's picture. We examine several modifications of the usual Ising model for which in certain limits a single-valued description becomes exact. Such models either exhibit no bulk critical behavior at all, even if the surface tension $\sigma$ vanishes, or have critical properties in a different universality class from the usual Ising-model (liquid-vapor) critical point.
\end{abstract}

\section{INTRODUCTION}

Interface Hamiltonians have proven very useful in understanding a wide variety of phenomena observed in coexisting phases, including roughening, ${ }^{1}$ layering, ${ }^{2,3}$ and wetting ${ }^{4,5}$ phase transitions. In an interface approach one considers only the degrees of freedom of a $(d-1)$ dimensional interface that is flat on macroscopic scales and whose vertical displacement from the flat reference plane specified by $z=0$ is given by a single-valued function $h(\mathbf{r})$ (in a continuum description). The energy for small amplitude and long-wavelength distortions of the interface can often be estimated from symmetry considerations and macroscopic thermodynamics; the appropriate interface Hamiltonian describing such distortions then takes on a particularly simple form. In the applications mentioned above, long-wavelength interface fluctuations play a crucial role in the phase transitions, which occur away from the bulk critical point, and approaches using interface Hamiltonians have yielded much insight. (See Binder, ${ }^{6}$ Jasnow, ${ }^{7}$ and $\mathrm{Zia}^{8}$ for recent reviews.)

In this paper we examine carefully the derivation and use of interface Hamiltonians and discuss their range of validity near the critical temperature $T_{c}$, concentrating for simplicity on the liquid-vapor interface and the Ising model. By "interface Hamiltonian" we mean a Hamiltonian that is a functional only of the single-valued function $h(\mathbf{r})$, where $\mathbf{r}$ is a $(d-1)$-dimensional vector. The interactions between different parts of the interface must be short ranged, decaying at least exponentially with distance. To achieve a simplified interfacial description, we must formally remove degrees of freedom present in the full $d$-dimensional Hamiltonian describing the two-phase system. As argued in more detail below, a simple interfacial description naturally arises on length scales large compared to the bulk correlation length $\xi_{B}$, since then the probability of finding bulk excitations ("bubbles" of the opposite phase) and multiple-valued interface configurations ("overhangs") [see Fig. 1(a)] is exponentially small. An equivalent interface description in which the integration over bulk degrees of freedom is explicitly carried out arises from the column model of Weeks. ${ }^{9}$ Here the volume of the system is divided into an array of columns with width $l \gg \xi_{B}$ and an integration over all degrees of freedom is carried out except for the average position of the interface $h(\mathbf{r})$ as determined from a fixed number of particles in each column.

The remaining long-wavelength interfacial degrees of freedom are accurately described by the simple quadratic "capillary-wave" Hamiltonian of Buff, Lovett, and Stillinger 10

$H_{\mathrm{CW}}=\int d^{d-1} \mathbf{r}\left[\frac{\sigma}{2}|\nabla h(\mathbf{r})|^{2}+\frac{1}{2} m g \Delta \rho h^{2}(r)\right]$,

where $\sigma$ is the surface tension, $\Delta \rho=\rho_{l}-\rho_{v}$ is the density difference between the liquid and the vapor, $m$ the molecular mass, and $g$ the gravitational acceleration. In terms of the Fourier series

$$
h(\mathbf{r})=\sum_{\mathbf{q}} \widetilde{h}(\mathbf{q}) e^{i \mathbf{q} \cdot \mathbf{r}},
$$

Eq. (1.1) takes on the simple form

$$
H_{\mathrm{CW}}=\frac{1}{2} \sigma L^{d-1} \sum_{\mathbf{q}} \widetilde{h}(\mathbf{q}) \widetilde{h}(-\mathbf{q})\left(q^{2}+L_{c}^{-2}\right),
$$


for a system with volume $L^{d}$ (which will tend to infinity), where the capillary length $L_{c}$ is defined as

$$
L_{c}=\left[\sigma / m g\left(\rho_{l}-\rho_{v}\right)\right]^{1 / 2} .
$$

Because of the restriction to long wavelengths implied by the coarse graining over length scales up to $l \gg \xi_{B}$, the sum over $q$ in (1.3) should be cut off at $|\mathbf{q}|=q_{\max }=\pi / l<<\pi / \xi_{B}$, consistent with the use of the macroscopic surface tension $\sigma$ in (1.1)-(1.4). The surface tension is not an analytic function of $\left(T_{c}-T\right)$ : the critical properties of $\sigma$ are determined by integrating over fluctuations of wavelengths less than and of order $\xi_{B}$ and obviously cannot be obtained from an analysis of (1.1).

Can one find an interfacial description valid also for fluctuations with wavelengths less than or of the order of $\xi_{B}$ ? If so, then one might be able to develop a theory for critical properties using a simple interface Hamiltonian. Wallace and $\mathrm{Zia}^{11}$ have suggested that this is possible using the nonlinear "drumhead" Hamiltonian

$H_{\mathrm{dh}}=\int d^{d-1} \mathbf{r}\left\{\sigma_{0}\left[1+|\nabla h(\mathbf{r})|^{2}\right]^{1 / 2}+\frac{1}{2} m g \Delta \rho h^{2}(r)\right\}$.

The square-root term gives the area of the distorted surface; macroscopic thermodynamic arguments suggest that (1.5) should be valid for large amplitude fluctuations in $h(\mathbf{r})$, in contrast to Eq. (1.1), which is truncated to lowest order in $|\nabla h|^{2}$. However, if $H_{\mathrm{dh}}$ is to be useful for short-wavelength distortions and to calculate critical properties, one must use some bare (unrenormalized) surface tension $\sigma_{0}$ rather than the macroscopic $\sigma$ which appears in (1.1). In $H_{\mathrm{dh}}$ the short-wavelength (large $q$ ) distortions are strongly coupled, and Wallace and $\mathrm{Zia}^{11}$ (see also Refs. 12-14) suggested that these short-wavelength modes could be controlled in a renormalization-group analysis without using a short-distance cutoff. From the analysis, formulated in terms of an expansion around $d=1$, they obtained a nontrivial fixed point with a critical exponent that they identified with the Ising bulk correlation length exponent $v$. In this picture, the interface is thought to become "fuzzy" over a length scale of $O\left(\xi_{B}\right)$ because of the incorporation of the fluctuations of a sharp interface over all momenta $q \gtrsim \xi_{B}^{-1}$.

We argue here that this picture is incorrect because bubble and overhang fluctuations (see Fig. 1) not describable in terms of a single-valued function $h(\mathbf{r})$ are an essential part of the physics on length scales less than and of order $\xi_{B}$. If one insists on describing the system in terms of fluctuating sharp interfaces even on these length scales then the function $h(\mathbf{r})$ necessarily becomes multiple valued due to bubbles and overhangs of size up to $O\left(\xi_{B}\right)$ which are present throughout the entire $d$-dimensional system. The interface Hamiltonian (1.5), based on a single-valued $h(\mathbf{r})$, suppresses all of these fluctuations. Because of this, we argue, any critical point obtained from such an interface Hamiltonian is in a different universality class from the usual Ising-model critical point. We demonstrate this explicitly for a particular twodimensional model (Sec. VII), but argue that it is true quite generally.

Bruce and Wallace and Schmittmann, ${ }^{15}$ in an $\epsilon=d-1$ expansion based not on a single-valued interface but on a more realistic droplet model, have argued that the differences between the Ising exponents and those obtained by Wallace and Zia's earlier expansion are due to dropletdroplet interactions and vanish as an essential singularity for $d \rightarrow 1$, so that the $\epsilon$ expansion may in fact be formally correct. The purpose of this paper is not to investigate the $\epsilon$ expansion but rather to discuss general aspects of and differences between systems with and without excitations like bubbles and overhangs. We argue that, notwithstanding the possible asymptotic equivalence of the critical exponents in the limit $d \rightarrow 1$, the "interface phenomenology"11 underlying Wallace and Zia's expansion that ignores bubbles and overhangs is not an accurate picture of the Ising critical behavior for any $d>1$.

In Sec. II we review the scaling theory of Widom, ${ }^{16,17}$ which relies on the similarity between bulk density fluctuations and fluctuations in the interfacial region to derive scaling laws relating the critical behavior of $\sigma$ to that of bulk thermodynamic properties. Although Widom's scaling theory for the interface is well known, its implications for questions concerning the range of validity of interface Hamiltonians have received less attention. Widom's theory suggests that no simplifications should arise when studying interfacial critical properties; the same fluctuations [bubbles on all length scales up to $O\left(\xi_{B}\right)$ ] which control bulk correlations are also relevant for interfacial critical properties.

However, Widom's picture ignores the long-wavelength interface distortions described by Eq. (1.1). Since for $g=0$ the energy of long-wavelength modes tends to zero as $q^{2}$, these modes are easily excited and cause a "wandering" of the interface. We examine in Sec. III the derivations of the interface Hamiltonians (1.1) and (1.5) and find that they are indeed valid, but only on sufficiently large length scales. The implications of interface wandering for the validity of Widom's picture and its role in critical phenomena are discussed in Sec. IV, following the ideas of Weeks. ${ }^{9}$ We conclude that the interface wandering occurring at length scales larger than $O\left(\xi_{B}\right)$ is unimportant in determining the critical behavior of the interface.

After these more general considerations regarding the connection between capillary waves and critical behavior, we turn to a more detailed discussion of $H_{\mathrm{dh}}$ and its underlying physics in Secs. V-VII. In Sec. V we focus on the drumhead Hamiltonian. We show that the restriction to a single-valued interface implies an asymmetry in the type of configurations that are taken into account, and that as a result the model does not exhibit the rotational symmetry found in fluids or an Ising model near $T_{c}$. (Similar conclusions have recently been reached independently by Teitel and Mukamel ${ }^{18}$ ). Although the lattice Ising-model Hamiltonian is not rotationally invariant, isotropy is obtained in the scaling limit $T \rightarrow T_{c}$ in that the interfacial free energy or surface tension, $\sigma(\theta)$, as a function of the interfacial orientation, $\theta$, vanishes as $T \rightarrow T_{c}$ for all orientations $\theta$. In contrast, the interfacial free energy of a drumhead model vanishes first at a particular orientation, while remaining nonzero at all other orientations. Thus the interfacial "stiffness," $\sigma(\theta)+d^{2} \sigma(\theta) / d \theta^{2}$, vanishes at $T_{c}$ for the Ising model but not for the drumhead model. As a result, the scaling behavior of the inter- 
face width obtained from the drumhead model is not in agreement with Widom's theory. In Sec. V we also briefly comment on the implications of these ideas for the validity of the $\epsilon=d-1$ expansion of the renormalization-group equations for the drumhead Hamiltonian, although our focus in this paper is not the technical validity of the $\epsilon$ expansion, but the general utility away from one dimension of the physical picture implied by the use of such an interface Hamiltonian.

One may also illuminate (Secs. VI and VII) the difficulties associated with using interface Hamiltonians for estimating bulk critical behavior by studying simple lattice models that interpolate between the Ising model and interface models in which the interfacial position $h(\mathbf{r})$ is single valued as in (1.1) and (1.5). The configurations with bubbles and overhangs may be suppressed in at least two possible ways. First, we can associate an extra energy with each interface segment that is oriented in a direction opposite to the macroscopic interfacial orientation. Such a modified Ising model has an appropriately defined surface tension whose vanishing is not connected with a bulk phase transition. This analysis (Sec. VI) also points out the shortcomings of the method of Müller-Hartmann and Zittart $z^{19}$ for calculating the surface tension of lattice models in a no-bubble, no-overhang approximation. The other way to suppress the bubbles and overhangs is to associate an extra energy, $E_{r}$, with the points (or lines in a three-dimensional system) where the interface reverses its orientation. Such a modification can be made explicitly in a two-dimensional Ising model, converting it into a still exactly solvable 8 -vertex model, ${ }^{20}$ as is shown in Sec. VII of this paper. As long as $E_{r}$ remains finite, the phase transition remains in the Ising universality class, albeit with a reduced critical region. However, in the limit $E_{r} \rightarrow \infty$, which is the limit in which one obtains a singlevalued interface on all length scales, the nature of the phase transition changes. This is due to the complete elimination of overhangs and bubbles, which are the dominant critical fluctuations for the Ising universality class of phase transition.

\section{WIDOM'S PICTURE OF THE CRITICAL INTERFACE}

Widom ${ }^{16,17}$ has generalized the classical theory of van der Waals to apply to the interface near the critical point. In this picture the distinction between bulk density fluctuations and interface inhomogeneities gradually disappears as the critical point is approached at coexistence. The underlying idea is that the bulk correlation length $\xi_{B}$ is the only important length scale determining the critical behavior. That is, $\xi_{B}$ is the basic length scale over which any density inhomogeneity extends, whether it arises from spontaneous density fluctuations in the bulk, or it represents the stable density gradient found at the liquidvapor interface. As $T \rightarrow T_{c}$, the interface width is thus $O\left(\xi_{B}\right)$ and its divergence is the same as that of $\xi_{B}$ $\left[\xi_{B} \sim\left(T_{c}-T\right)^{-v}\right]$. These ideas lead at once to scaling laws ${ }^{16,17}$ for $\mu$, the critical exponent which describes how the surface tension $\sigma$ vanishes as $T_{c}$ is approached $\left[\sigma \sim\left(T_{c}-T\right)^{\mu}\right]$. Since $\sigma$ is the excess free energy per unit area of the interface, the divergence of the interface width as $\xi_{B}$ shows that $\sigma \xi_{B}^{-1}$ is proportional to the excess free energy per unit of volume in the interfacial region. The assumption that the inhomogeneities at the surface become more and more like bulk critical fluctuations then dictates that $\sigma \xi_{B}^{-1}$ vanishes as the singular part of the bulk free-energy density. Hence $\sigma \xi_{B}^{-1} \sim\left(T_{c}-T\right)^{2-\alpha}$, or

$$
\mu+v=2-\alpha \text {. }
$$

The Widom scaling law

$$
\mu=(d-1) v
$$

follows in a similar way from the assumption that fluctuations in volumes of size $\xi_{B}^{d}$ represent essentially independent elementary excitations, so that $\sigma \xi_{B}^{d-1} \sim k_{B} T$.

Equation (2.2) has received experimental conformation (see, e.g., Refs. 7 and 17), and all known results for $\mu$ from exact solutions of lattice models for $d \leq 4$ are in agreement with the above scaling laws. They have also been verified to first order in a $\epsilon=4-d$ expansion ${ }^{21,22}$ [because of the breakdown of hyperscaling in $d>4,(2.2)$ ceases to hold above four dimensions]. The validity of the scaling laws and by implication the fundamental correctness of Widom's physical picture below four dimensions thus appears to be amply confirmed. Clearly this picture relies on the similarity of interface and bulk critical fluctuations and holds little hope for establishing a simplified interfacial theory of critical phenomena.

\section{EFFECTIVE INTERFACE HAMILTONIAN: LONG-WAVELENGTH PICTURE}

However, the above arguments have not taken account of interface fluctuations at wavelengths much larger than $\xi_{B}$ as described by Eq. (1.1). The consequences of these fluctuations are discussed in this section. We first consider several derivations leading to well-defined interface Hamiltonians and then examine their implications for the behavior of the interface and for the validity of the Widom $^{16,17}$ picture. We consider an Ising system in zero field, which has a critical point in the same universality class as the liquid-vapor system.

The first step towards the derivation of an effective interface Hamiltonian near the critical point is the standard coarse graining of the Ising spins over some length scale greater than microscopic scales, but much less than $\xi_{B}$, leading to the Landau-Ginzburg-Wilson ${ }^{23,24}$ Hamiltonian for the spin field $\phi$,

$$
H_{\mathrm{LGW}}=\int d^{d} \mathbf{R}\left[\frac{1}{2}|\nabla \phi|^{2}+V(\phi)\right]
$$

with

$$
V(\phi)=\frac{1}{2} \tau \phi^{2}+\frac{1}{4} u \phi^{4} .
$$

Below $T_{c}$, we have $\tau<0$ so that $V(\phi)$ has two minima at $\phi=\phi_{ \pm}= \pm(|\tau| / u)^{1 / 2}$ associated with the up $(+)$ and down $(-)$ states of the Ising spins. Nonuniform boundary conditions that favor the $(+)$ phase at the bottom of the system and the $(-)$ phase at the top are used to force an interface into the system (see Fig. 1). The interface free energy is proportional to the logarithm of the ratio of $Z_{++}$, the partition function of the system with uniform ++ boundary conditions, and $Z_{+-}$, the partition func- 
tion of the system with nonuniform +- boundary conditions. ${ }^{25,26}$

Of course, most of the bubbles of overturned spins and the overhangs at the interface are still manifest in $H_{\mathrm{LGW}}$, since at this stage the coarse graining involves only length scales small compared to $\xi_{B}$. This is illustrated in Fig. 1 (b), where we have qualitatively sketched the remaining bubbles and overhangs in the coarse-grained field $\phi$ associated with the configuration of Fig. 1(a). As a result, $H_{\text {LGW }}$ is still a full $d$-dimensional object, even for a system with an interface. Next, let us integrate out more short-wavelength fluctuations until we have reached a length scale $l \gg \xi_{B}$. At this stage the probability of finding bubbles and overhangs is exponentially small and the

(a)

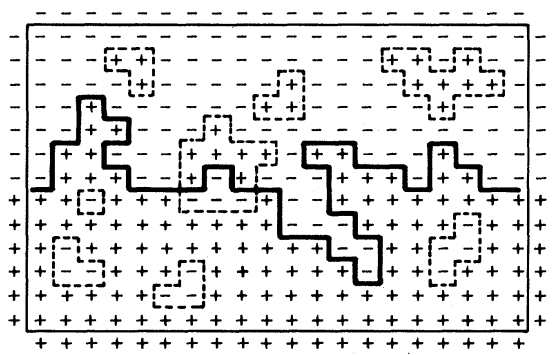

(b)

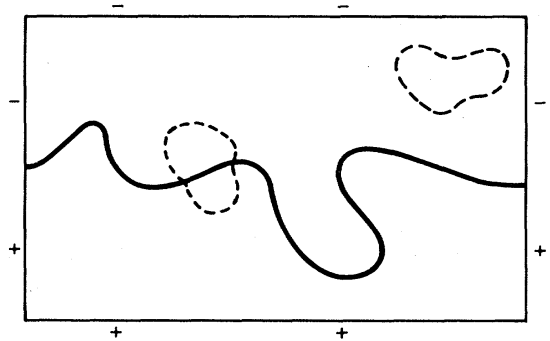

(c)

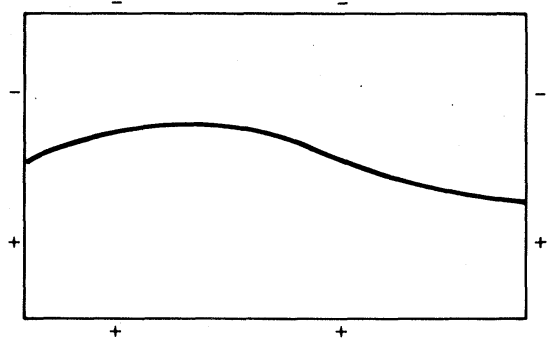

FIG. 1. Typical configuration of an Ising model with an interface viewed on different length scales. Note the use of $(+-)$ boundary conditions to enforce the existence of an interface. (a) The Ising model seen on the scale of the square lattice has several bubbles (dashed lines) and a "long contour" with overhangs (solid line), which extends from one side of the system to the other, separating + and - spins. Note the ambiguity in the choice of the solid line in case a bubble intersects the long contour. (b) The interface and bubbles in the field configuration of $H_{\mathrm{LGW}}$, obtained by coarse graining of (a) on a length scale less than $\xi_{B}$. The largest bubbles and overhangs in (a) have survived the coarse graining. (c) The long wavelength picture of the interface obtained by integrating over the critical length scales less than and of order $\xi_{B}$ in (b). Essentially all bubbles have disappeared and a single-valued interface remains. remaining fluctuations in the system are fairly accurately described in terms of a single-valued nearly flat $(d-1)$ dimensional interface, as illustrated in Fig. 1(c). The remaining small distortions away from the average interface position with $h(\mathbf{r})=0$ can be described by the $(d-1)$-dimensional capillary-wave interface Hamiltonian

$$
H_{\mathrm{CW}}=\int d^{d-1} \mathbf{r} \frac{1}{2} \sigma|\nabla h(\mathbf{r})|^{2},
$$

in which the interfacial free-energy parameter (surface tension) $\sigma$ results from the integration over the shorter length scales, which include the relevant ones up to order $\xi_{B}$. Strictly speaking, the surface tension $\sigma$ that enters in (3.3) should be $\sigma(l)$, the surface tension renormalized only out to length scale $l$. However, for $l \gg \xi_{B}$, the difference between $\sigma(l)$ and the macroscopic surface tension $\sigma$ is of relative order $\left(\xi_{B} / l\right)^{d-1} \ln \left(l / \xi_{B}\right)$ and thus is small. ${ }^{9}$

Of course, $\frac{1}{2} \int d^{d-1} \mathbf{r}|\nabla h|^{2}$ is only to lowest order equal to the change in area of the Gibbs dividing surface, and macroscopic thermodynamics or rotational invariance suggest that the "drumhead" interface Hamiltonian of the form

$$
H_{\mathrm{dh}}=\int d^{d-1} \mathbf{r} \sigma\left[1+|\nabla h(\mathbf{r})|^{2}\right]^{1 / 2}
$$

would be accurate for larger amplitude distortions in $h(\mathbf{r})$. However, as shown in the Appendix, where the meanfield-type derivations of $H_{\mathrm{dh}}$ from $H_{\mathrm{LGW}}$ are discussed, the validity of both $H_{\mathrm{CW}}$ and $H_{\mathrm{dh}}$ is limited to length scales large enough that $\left\langle|\nabla h|^{2}\right\rangle$ is small compared to unity. Under these circumstances the higher-order terms in $|\nabla h|^{2}$ are even smaller and make little difference in evaluating the partition function (recall that the interface is parallel to the reference frame $z=0$ on a macroscopic scale). Both Hamiltonians describe single-valued interfaces and hence have no room for information about important pieces of the short-scale physics, namely overhangs and bubbles. Extrapolating backwards by taking $H_{\mathrm{dh}}$ literally on all length scales and using some bare $\sigma_{0}$ cannot properly "undo" the previous coarse graining.

Perhaps this point can be clarified if we consider the column model of Weeks, ${ }^{9}$ where the integration over bulk degrees of freedom is explicitly carried out. This procedure can be used to derive formally a single-valued but generally very complicated interface Hamiltonian valid on arbitrarily small length scales for, say, a liquid-vapor interface. We begin by dividing the volume of the system up into columns of width $w$ and infinite height (for a finite system the height is taken equal to the system size $L$ ). In each column a variable $h_{i}$ is defined as the location of the local Gibbs dividing surface ${ }^{9}$ defined in terms of the number of particles in that column. Then an integration over all degrees of freedom with fixed set of heights $\left\{h_{i}\right\}$ yields an interface Hamiltonian $H_{w}\left(\left\{h_{i}\right\}\right)$ which might be used, in principle, to calculate the bulk critical behavior of the system.

However, if $w$ is a fixed length (which does not scale with $\left.\xi_{B}\right), H_{w}\left(\left\{h_{i}\right\}\right)$ will have intercolumn interactions over a longer and longer range of $O\left(\xi_{B}\right)$ as $T \rightarrow T_{c}$. These short-wavelength interactions over distances less than or of order $\xi_{B}$ will be very complicated, and will contain information about bulk critical behavior-they 
cannot be expected to be of a form that reduces to $\sigma_{0}\left(1+|\nabla h|^{2}\right)^{1 / 2}$ in the continuum limit. (In fact, it is conceivable that the parameters themselves have singular functional dependences on the bare parameters and the temperature as a result of the integration over the infinite column heights.) On the other hand, for large column widths $w>\xi_{B}$, we do arrive at the simple interface Hamiltonian (1.1), but one where the effects of bulk critical fluctuations have already been integrated out. ${ }^{9}$ This leads to singular behavior of $\sigma(T)$ as $T \rightarrow T_{c}$.

\section{INTERFACE WANDERING IN CAPILLARY-WAVE THEORY}

Having established the validity of $H_{\mathrm{CW}}$ at sufficiently large length scales, we discuss briefly its implications for the Widom picture, where long-wavelength interface fluctuations (capillary waves) are ignored. It is well known ${ }^{10}$ that $H_{\mathrm{CW}}$ predicts a "wandering" of the interface for $d \leq 3$, which leads to a divergence of the interface width in an infinite system as $g \rightarrow 0^{+}$. Interface wandering also plays a major role in Wallace and Zia's ${ }^{11}$ approach. At first glance, capillary waves seem to invalidate Widom's ${ }^{16,17}$ picture of an intrinsic interface whose width is $O\left(\xi_{B}\right)$.

However, as argued by Weeks, ${ }^{9,27}$ there are really two different measures of the interface width, only one of which corresponds to Widom's intrinsic width. Moreover, it is found that $H_{\mathrm{CW}}$ is consistent with the proper scaling relations if a short-wavelength cutoff at length $l=N \xi_{B}$ is used with $N$ a fixed number $\gg 1$. Since for extracting powers the precise value of $N$ is immaterial, we will henceforth, for convenience, take $N$ equal to unity. To obtain the scaling behavior, consider the height difference correlation function calculated using $H_{\mathrm{CW}}$ in Eq. (1.1):

$$
\begin{aligned}
G(r) & \equiv \frac{1}{2 L^{d-1}} \int d \mathbf{s}\left\langle[h(\mathbf{r}+\mathbf{s})-h(\mathbf{s})]^{2}\right\rangle \\
& \approx \frac{1}{\beta \sigma(2 \pi)^{d-1}} \int_{|q|<\pi / \xi_{B}} d^{d-1} \mathbf{q} \frac{1-e^{i \mathbf{q} \cdot \mathbf{r}}}{q^{2}+L_{c}^{-2}},
\end{aligned}
$$

where we have taken the infinite volume limit in Eq. (4.2). Long-wavelength fluctuations between regions of the interface separated by distances much greater than $\xi_{B}$ cause the total interface width $W_{\infty}^{2} \equiv G(\infty)$ calculated from (4.2) to diverge as $L_{c} \rightarrow \infty(g \rightarrow 0)$ for $d \leq 3$. To see this, note that the effect of a large but finite $L_{c}$ can be approximated by a small wave-vector cutoff $q_{\mathrm{min}} \simeq \pi / L_{c}$ in (4.2) (such a cutoff at $q_{m} \simeq \pi / L$ would also give the effect of finite system size $L^{d}$ ) and we find

$$
\begin{aligned}
W_{\infty}^{2} \approx & \frac{1}{\beta \sigma(2 \pi)^{d-1}} \int_{\pi / L_{c}<q<\pi / \xi_{B}} d^{d-1} \mathbf{q} q^{-2} \\
= & \frac{2}{\beta \sigma(4 \pi)^{d-1 / 2} \Gamma((d-1) / 2)(d-3)} \\
& \times\left[\left(\pi / \xi_{B}\right)^{d-3}-\left(\pi / L_{c}\right)^{d-3}\right] .
\end{aligned}
$$

Thus $W_{\infty}^{2}$ is proportional to $L_{c}^{3-d}$ for $d<3$, to $\ln L_{c}$ for $d=3$, and is finite and independent of $L_{c}$ as $L_{c} \rightarrow \infty$, for $d>3$.
However, Widom's picture concentrates on the important fluctuations determining bulk critical properties with wavelengths less than or of order $\xi_{B}$ and ignores the effects of the longer-wavelength fluctuations which give rise to the divergences in (4.4). An estimate of the interface width when these long-wavelength fluctuations are suppressed can be made by considering fluctuations between regions of the interface separated by distances of $O\left(\xi_{B}\right)$ (in general, of order $N \xi_{B}$ ), the minimum distance for which $H_{\mathrm{CW}}$ can be trusted, and the range over which the elementary density fluctuations should occur., ${ }^{9,27}$ Thus defining the local width $^{9,27,28} W_{\xi_{B}}^{2} \equiv G\left(\xi_{B}\right)$, we find from Eq. (4.2), after using the Widom scaling relation $\beta \sigma \xi_{B}^{d-1} \approx$ const, that $W_{\xi_{B}} \sim \xi_{B}$ as $T \rightarrow T_{c}$ for all $d<4$ independent of $L_{c}$. Thus the local width behaves in just the way envisioned by Widom. ${ }^{16,17}$ Further, as mentioned in Sec. III, the longer-wavelength fluctuations in $H_{\mathrm{CW}}$ carry very little free energy ${ }^{9,27}$ and can be ignored in considering the singular behavior of $\sigma$ near $T_{c}$. This confirms the essential validity of the Widom picture for the relationship between bulk and surface critical properties.

Another way of arguing for the irrelevance of interface wandering for critical behavior is to compute the fraction $f$ of the volume of the system which is influenced by interface fluctuations. If we consider a finite system of size $L^{d}$ with $g=0$, we can use Eq. (4.4) to estimate the size of the region affected by interface fluctuations provided we replace $L_{c}$ by $L$. We therefore find from (4.4)

$$
f \equiv \frac{L^{d-1} W_{\infty}}{L^{d}} \propto \frac{1}{\Gamma((d-1) / 2) L^{(d-1) / 2}}, \quad 1<d<3 .
$$

Thus interface fluctuations affect an arbitrarily small fraction of the bulk for all $d$ with $1<d<3$ in the thermodynamic limit. $^{29}$ Further, the wandering occurs for all temperatures less than $T_{c}$.

It is sometimes argued ${ }^{11,14}$ that capillary waves are the driving force that causes $T_{c}$ to tend to zero as $d \rightarrow 1$, in analogy with the suppression of $T_{c}^{\prime}$ to zero by spin waves in the Heisenberg model as $d \rightarrow 2$. This analogy, discussed further in Sec. V, was exploited by Wallace and $\mathrm{Zia},{ }^{11}$ who expanded their renormalization-group equations in powers of $d-1$. Indeed, interface fluctuations increase as $d$ is decreased, but near $d=1$, Eq. (4.5) can be written as

$$
f=\frac{W}{L} \propto(d-1) L^{(1-d) / 2},
$$

where we have used the fact that $1 / \Gamma(x)=x+O\left(x^{2}\right)$. If one considers a finite system in $d$ dimensions with the interface fixed on only one side (this eliminates the trivial $k=0$ translation mode of the interface as a whole, which is even present in a system without capillary waves ${ }^{9,30}$ ), the interface fluctuations on the other side due to capillary waves are of the order of $W$, justifying its interpretation as the interface width. According to Eq. (4.6), $W / L$ actually decreases with decreasing $d$ when $d-1$ becomes less than some value of order $(\ln L)^{-1}$. This can be interpreted as a gradual stiffening of the interface in the limit $d \rightarrow 1$, which arises from the reduction in phase space 
available for the interface fluctuations as the interface dimension tends to zero. However, it is above all a warning that the behavior near $d=1$ is quite singular and that results depend sensitively on the order of the limits $d \rightarrow 1$ and $L \rightarrow \infty$.

The suppression of $T_{c}$ to zero in the limit $d \rightarrow 1$ is much more naturally attributed to bulk fluctuations. Overturning Ising spins in an area of size $l$ in $d$ dimensions results in an energy increase proportional to $l^{d-1}$; for $d=1$ this energy is independent of $l$ and so at any nonzero temperature arbitrarily large bulk excitations can be created. The fact that $T_{c} \rightarrow 0$ for $d \rightarrow 1$ follows immediately from such considerations, and should not be attributed to an increased activity of capillary waves near $d=1$.

An indirect experimental test for the interface picture described in the preceding three sections is possible by comparison of the results of light scattering experiments on fluid interfaces near the critical point with the theoretical predictions of Jasnow and Rudnick, ${ }^{31}$ who include both the contributions from the long-wavelength capillary waves and those from the "intrinsic profile." The experimental data of $\mathrm{Wu}$ and $\mathrm{Webb}^{32}$ are in good agreement with this theory, and indeed show a changeover ${ }^{33}$ in behavior at wavelengths of the order of $\xi_{B}$.

\section{CRITIQUE OF THE DRUMHEAD MODEL}

In this section we wish to elaborate further on why the drumhead Hamiltonian (3.4) cannot give a correct description of the behavior of an interface near the bulk critical temperature. Of course, the drumhead Hamiltonian by itself has the full rotational invariance of $d$ dimensional space, because the energy is simply proportional to the area of the interface. However, the restriction that $h(\mathbf{r})$ is a single-valued function clearly breaks this rotational symmetry by making the $z$ axis special in the sense that only configurations without overhangs and bubbles with respect to this particular axis are taken into account in evaluating the partition function. Therefore, the surface tension $\sigma(\theta)$ of the drumhead model will depend on the tipping angle $\theta$ at any nonzero temperature, although it is independent of $\theta$ at $T=0$, where there are no fluctuations (cf. Ref. 18). Of course, the Ising model is well known to exhibit isotropic scaling properties near $T_{c}$ even in the presence of nonuniform boundary conditions, and despite the lack of complete rotational invariance of the microscopic lattice Hamiltonian; these differences become irrelevant near $T_{c}$. For an interface approach to preserve a similar rotational invariance in the scaling limit, one must average over an isotropic set of configurations and allow $h(\mathbf{r})$ to be multiple valued. If this is indeed allowed then the interface will form bubbles and overhangs on length scales less than or of order of the bulk correlation length $\xi_{B}$. These bubble and overhang fluctuations are an essential part of the critical Isingmodel universality class. The restriction to single valuedness in the drumhead model completely suppresses these important fluctuations in a fashion that breaks rotational symmetry. Teitel and Mukamel ${ }^{18}$ have explicitly shown that the free energy associated with $H_{\mathrm{dh}}$ is not isotropic to $O(T)$ in any dimension $d \neq 1$. This means that the simple square-root form of the drumhead Hamiltonian will not be preserved under a renormalization-group rescaling when the short-distance cutoff is less than or of order $\xi_{B}$. Rotational invariance is not restored in the scaling limit, as is shown explicitly below for $d=2$.

Up to now, we have not addressed the question of whether the interface approach to bulk critical phenomena could be asymptotically correct near $d=1$, since our main interest is in the general validity of the interface picture, in particular for $d>1$. Although our analysis, particularly in Sec. VII, shows that this approach fails in $d=2$ due to the relevance of bubbles and overhangs, it does not assess the behavior in the limit $d \rightarrow 1$. The latter limit, though rather singular, is of interest because the explicit calculations of Wallace and $\mathrm{Zia}^{11}$ were based on a $\epsilon=d-1$ expansion of the renormalization-group equations for $H_{\mathrm{dh}}$. A justification for such an expansion comes from the recent work of Bruce and Wallace, ${ }^{15}$ who have carried out an $\epsilon=d-1$ expansion of a droplet model. Such droplet models were first proposed for general dimension by Fisher. ${ }^{34}$ While not taking account of overhangs in the surface of each droplet or of direct interactions between droplets, Bruce and Wallace ${ }^{15}$ do consider some multiple droplet effects; they argue that the correlation-length exponent $v$ and the order-parameter exponent $\beta$ at the Ising critical point are separately determined by two different mechanisms for small $\epsilon=d-1$, since then the droplet boundaries are dilute even at $T_{c}$. Within their approach, $v$ is determined by droplet or interface fluctuations and its $\epsilon$ expansion is the same as the one of Wallace and $\mathrm{Zia},{ }^{11}$ while quantities like $\beta$ that reflect the droplet density vanish to all orders in a powerseries expansion in $\epsilon$. Instead, $\beta$ has an essential singularity for $\epsilon \rightarrow 0$ of the form $\beta \propto \exp (-2 / \epsilon) .{ }^{35}$ Presumably the other critical exponents also have essential singularities if multiple droplet and overhang effects are taken into account.

The work of Bruce and Wallace ${ }^{15}$ shows that the $\epsilon$ expansion of Wallace and $\mathrm{Zia}^{11}$ may give the correct expansion for the true Ising exponents since bubbles and overhanges contribute only in order $\exp (-c / \epsilon)$ near $d=1$, with $c$ of order unity, which means that they do not contribute at all to the formal perturbation expansion. A likely scenario that reconciles the conclusions of Bruce and Wallace ${ }^{15}$ with our picture is the following: although bubbles and overhangs remain relevant for all $d>1$ at the fixed point studied by Wallace and $\mathrm{Zia}$, the proper Ising fixed point (which includes overhangs and bubbles) moves close to it in the limit $d \rightarrow 1$. In this limit, the exponents at the two fixed points become asymptotically identical. ${ }^{36}$ If this is indeed the case, we expect the two fixed points to move rapidly apart for increasing $d$, since the physics they describe is very different. By the time we arrive at $d=2$ the exponents at the two fixed points presumably differ by order unity.

We do find some arguments that have been presented for the validity of the $\epsilon=d-1$ expansion less than compelling. For example, it has been suggested that the $\epsilon=d-1$ expansion derived from the drumhead Hamiltonian is similar in spirit to the $\epsilon=d-2$ expansion for $n \geq 2$ Heisenberg spin models. ${ }^{37}$ We believe that the anal- 
ogy between the capillary waves in the former and the spin waves in the latter is rather weak. The divergent fluctuations of the spin waves are known to play an essential role in the disordering of the two-dimensional $n \geq 2$ spin models. In the one-dimensional Ising model, however, capillary waves do not even exist. The bulk fluctuations, namely bubbles, which cause the disordering of the $d=1$ Ising model are not included in the $\epsilon=d-1$ expansion of Wallace and Zia.

Let us now consider the drumhead model in two bulk dimensions. ${ }^{38}$ We must have a short-distance cutoff for the model to be well defined; for convenience, we will set it on a lattice, since we do not expect this to affect the scaling behavior, just as is the case for the Ising model near $T_{c}$. The Hamiltonian is then ${ }^{39}$

$$
H_{\mathrm{ldh}}=\sum_{i} \sigma_{0}\left[1+\left(h_{i}-h_{i+1}\right)^{2}\right]^{1 / 2}
$$

where the heights $\left\{h_{i}\right\}$ are integers. The interfacial free energy, $\sigma$, as obtained from this model is simply

$$
\exp (-\beta \sigma)=\sum_{n=-\infty}^{\infty} \exp \left[-\beta \sigma_{0}\left(1+n^{2}\right)^{1 / 2}\right]
$$

In the limit $T \rightarrow 0(\beta \rightarrow \infty)$, we have $\sigma=\sigma_{0}$. As the temperature is increased, $\sigma$ decreases, eventually vanishing at some temperature $T_{0}$ as

$$
\sigma \sim\left(T_{0}-T\right)^{\mu}, \mu=1
$$

The Ising model also has surface tension exponent $\mu=1$, so (5.3) could be taken as a success of the drumhead model, but the result is obtained only because $\sigma$ is a smooth, monotonically decreasing function of $T$ which obviously vanishes with a finite, negative derivative $d \sigma / d T$. This argument is generalized in the next section.

Since the difference variables $h_{i}-h_{i+1}$ are independent, the height difference correlation function (4.1) for the present lattice drumhead model (5.1) is the analog of the time correlation function in a one-dimensional ( $1 d$ ) random walk. It is given by

$$
\begin{aligned}
G(r) & \equiv \frac{1}{2}\left\langle\left(h_{i}-h_{i+r}\right)^{2}\right\rangle=r G(1) \\
& =\frac{r}{2} e^{\beta \sigma_{0}} \sum_{n=-\infty}^{\infty} n^{2} \exp \left[-\beta \sigma_{0}\left(1+n^{2}\right)^{1 / 2}\right] .
\end{aligned}
$$

The nearest-neighbor mean-square height difference is $2 G(1)$ and is a smooth monotonically increasing function of $T$ that is finite for all finite temperatures. Using (5.4), we may now illustrate some differences between the Ising critical behavior and the behavior of the lattice drumhead Hamiltonian at its "critical temperature" $T_{0}$, defined by the vanishing of $\sigma$.

One implication of the isotropic scaling behavior at the critical point of the Ising model is that the interfacial tension vanishes for all possible interface orientations. For the drumhead model this is not the case. The interfacial tension $\sigma(\theta)$, as a function of the angle $\theta$ between the normal to the interface and the $z$ axis, has a minimum at $\theta=0$. For any nonzero temperature the interfacial tension increases with $\theta^{2}$, due to the restriction of $h(\mathbf{r})$ to single valuedness. In two bulk dimensions, the height difference correlation function at long distances $r$ becomes, accord- ing to thermodynamic fluctuation theory,

$$
\begin{aligned}
G(r) & \equiv \frac{1}{2}\left\langle[h(0)-h(r)]^{2}\right\rangle \\
& \simeq \frac{\frac{1}{2} \int_{-\infty}^{\infty} d h h^{2} \exp [-\beta L \sigma(\theta)]}{\int_{-\infty}^{\infty} d h \exp [-\beta L \sigma(\theta)]},
\end{aligned}
$$

where $L=\left(h^{2}+r^{2}\right)^{1 / 2}$ and $\tan \theta=h / r$. In the limit of large $r$, a steepest-descent calculation yields

$$
G(r) \simeq r k_{B} T / 2\left(\sigma+\sigma^{\prime \prime}\right),
$$

where $\sigma^{\prime \prime}=d^{2} \sigma(\theta) /\left.d \theta^{2}\right|_{\theta=0}$. Comparing (5.4) and (5.6) we find that the so-called surface stiffness ${ }^{30} \sigma+\sigma^{\prime \prime}$ does not vanish in the lattice drumhead model at any finite temperature. This should be contrasted with the twodimensional Ising model, where $\sigma+\sigma^{\prime \prime}$ does vanish as $T_{c}$ is approached from below. This shows that the restriction to a single-valued interface height has produced a serious violation of the rotational symmetry expected near $T_{c}$.

From the "random-walk interpretation" of (5.4) (which carries over to the continuum case), it is also clear that the lattice drumhead model does not obey the scaling law (2.2). If we define the local interfacial width as $W_{\xi_{B}}=\left[G\left(\xi_{B}\right)\right]^{1 / 2}$, as discussed in the preceding section, then we find

$$
W_{\xi_{B}} \sim \xi_{B}^{1 / 2}
$$

at the "critical" point $T_{0}$ of the lattice drumhead model where $\sigma$ vanishes but $\sigma^{\prime}$ ' remains finite. By the Widom scaling law (2.2) we would expect, however, $W_{\xi_{B}} \simeq \xi_{B} \propto\left(T_{0}-T\right)^{-1}$. Thus the interfacial width, which is a length normal to the interface, has a different critical exponent than the correlation length. Such anisotropic scaling is very different from the isotropic scaling behavior found at the $d=2$ Ising-model critical point. As discussed in Sec. IV, capillary-wave theory using the proper macroscopic surface tension is in general consistent with Widom's scaling theory; the reason the different result (5.7) arises in the lattice drumhead model can be traced back to the nonvanishing of the interfacial stiffness $\sigma+\sigma^{\prime \prime}$ in that model. We believe that this nonvanishing of the interfacial stiffness and the resulting anisotropic scaling will occur in the drumhead model with a short-distance cutoff in any bulk dimension $d>1$.

\section{DERIVATIONS OF AN EFFECTIVE INTERFACE HAMILTONIAN: MODIFIED ISING MODELS}

In this section we describe two limits in which the interface position $h(\mathbf{r})$ of an Ising model becomes single valued on all length scales, in order to clarify the difference between the behavior of systems exactly described by interface Hamiltonians such as $H_{\mathrm{CW}}$ and $H_{\mathrm{dh}}$ and that of the ordinary Ising model near criticality. The necessity to introduce, to this end, severe modifications into the Ising model, can be understood from the low-temperature picture of the interface. At sufficiently low $T$, it is clear that the Ising interface is indeed represented by a single-valued function without overhangs. After properly incorporating the anisotropy in the surface tension, the drumhead or capillary-wave Hamiltonians accurately describe long- 
wavelength distortions. ${ }^{40,41}$ From a renormalizationgroup point of view, this statement is equivalent to our previous contentions that at long wavelengths we arrive at the capillary-wave Hamiltonian, since on rescaling lengths the renormalization flow for $T<T_{c}$ is towards the $T=0$ fixed point. In order to extend the validity of this type of Hamiltonian to shorter length scales and higher temperatures, the bulk density fluctuations (bubbles) and overhangs must be suppressed. This can be achieved by taking one of the interaction parameters to be infinitely large.

\section{A. Anisotropic Ising model in SOS limit}

Consider an Ising model with an interface on a hypercubic lattice with ferromagnetic bonds $J_{\perp}$ perpendicular to the interface and bonds $J$ in the other directions. Since every overhang and bubble creates extra broken $J_{\perp}$ bonds, they can be suppressed by increasing $J_{\perp}$. Accordingly in the limit $J_{\perp} \rightarrow \infty$ only a single-valued solid-on-solid (SOS) interface survives. Each state of the system can then be characterized by the set of heights $\left\{h_{i}\right\}$ which give the interface location in each column, just as in Weeks's column method, ${ }^{9}$ though here the columns are of microscopic width. The resulting $(d-1)$-dimensional interface Hamiltonian has short-range interactions of the form $2 J\left|h_{i}-h_{j}\right|(i, j$ nearest neighbors) which resemble the discrete version $^{39}$ of the drumhead Hamiltonian

$$
2 J \sum_{i}\left[1+\sum_{\alpha}\left(\Delta_{i \alpha} h_{i}\right)^{2}\right]^{1 / 2}
$$

(here $\Delta_{i \alpha}$ is the discrete gradient operator, discussed for $d=2$ in the preceding section).

For large $J_{\perp}$, the interfacial free energy of the anisotropic Ising model, $\sigma\left(J_{\perp}, J, T\right)$ may be separated into the energy, $2 J_{1}$, of the flat $T=0$ interface and the remainder, which is due to the interfacial wandering at $T \neq 0$. This latter term will be independent of $J_{\perp}$ in the limit $J_{\perp} \rightarrow \infty$ :

$$
\lim _{J_{\perp} \rightarrow \infty}\left[\sigma\left(J_{\perp}, J, T,\right)-2 J_{\perp}\right]=f(J, T) .
$$

The function $f(J, T)$ arises from the intercolumn energies $2 J\left|h_{i}-h_{j}\right|$ and the entropy of interfacial configurations with overhangs and bubbles forbidden. Thus the interfacial free energy in the corresponding SOS model is precisely

$$
\sigma^{\mathrm{SOS}}\left(J_{\perp}, J, T\right)=2 J_{\perp}+f(J, T) .
$$

Since the identification of $\sigma$ and $\sigma^{\mathrm{SOS}}$ is correct only in the limit $J_{1} \rightarrow \infty$, where, from (6.2), $\sigma^{\text {sos }} \rightarrow \infty$, the SOS model cannot be used to study the critical point $\sigma \rightarrow 0$. This is consistent with the fact that $T_{c} \rightarrow \infty$ in this limit also, due to the suppression of the bulk excitations.

Now consider the approximation for the isotropic Ising model, obtained by replacing $J_{\perp}$ by $J$ in $\sigma^{\text {sos }}$

$$
\sigma(J, J, T) \approx \sigma^{\operatorname{sos}}(J, J, T) \text {. }
$$

This approximation gives the correct $T=0$ energy. Moreover, the first few terms in a low-temperature expan- sion for $\sigma$ and $\sigma^{\text {SOS }}$ are identical, since the overhangs and bubbles have a higher excitation energy than the lowestenergy surface excitations. This approximation does yield a $\sigma$ that vanishes at some temperature $T_{c}^{\mathrm{SOS}}$. However, since $f$ is a smooth function, $\sigma$ in (6.3) cannot have a singularity for $T \rightarrow T_{c}^{\mathrm{SOS}}$. For the exponent $\mu^{\mathrm{SOS}}$,

$$
\sigma^{\mathrm{SOS}} \sim\left(T_{c}^{\mathrm{SOS}}-T\right)^{\mu^{\mathrm{SOS}}},
$$

one therefore always obtains the value unity in this approximation. The neglect of bulk excitations and overhangs eliminates the possibility of a singularity in $\sigma^{\mathrm{SOS}}$.

This approximate procedure happens to give the exact answer $\mu=1$ when applied to the $2 d$ Ising model $^{19,39}$ (cf. Sec. V). For the square lattice nearest-neighbor model with an interface parallel to one of the lattice directions, it even gives the exact result for $\sigma$ due to a fortuitous cancellation of the contributions from bubbles and overhangs. Such a cancellation does not occur for interfaces tilted at some nonzero angle, ${ }^{42,43}$ however, and cannot be expected in general.

Müller-Hartmann and Zittartz ${ }^{19}$ have applied approximation (5.3) to the antiferromagnetic Ising model in a field, ${ }^{44}$ and several other workers ${ }^{45-54}$ have used the method in a variety of models. Because the first few terms in a low-temperature expansion are correct in this approach, it can sometimes yield a rather accurate estimate ${ }^{55}$ for $T_{c}$ [as obtained from solving $\sigma^{\mathrm{SOS}}(J, J, T)=0$ ], especially if $\mu$ is a priori known to be close to 1 . In most cases studied, this interface method is only exact in the limit where some energy $J_{1} \rightarrow \infty$, leading to a suppression of all bulk excitations and causing $T_{c} \rightarrow \infty$. Obviously, one therefore gains no insight into bulk critical phenomena by the artifice of studying such an interface model.

\section{B. Modified Ising model}

A different modification of the Ising model on a hypercubic lattice can lead to an exact interfacial Hamiltonian (SOS model) but still allow the interfacial free energy to remain finite and vanish at some temperature. Thus let us add to the Hamiltonian of the usual nearest-neighbor ferromagnetic Ising model with coupling $J$ the additional interaction

$$
\frac{J_{1}}{4} \sum_{\mathbf{R}}[1-s(\mathbf{R})][1+s(\mathbf{R}+a \hat{z})],
$$

where $a \hat{z}$ is the nearest-neighbor vector parallel to the $z$ axis and pointing "upwards." This term gives an additional energy of $J_{1}$ for each nearest-neighbor pair of spins in which $s(\mathbf{R})=-1$ and $s(\mathbf{R}+a \hat{z})=+1$. We now have the possibility of two different kinds of interfaces running normal to the $z$ axis: If the $s=-1$ phase is above the $s=+1$ phase, the ground-state energy of the interface per column of spins (per unit area) is

$$
\sigma_{+}^{-}(T=0)=2 J \text {, }
$$

while when the $s=+1$ phase is above the energy is

$$
\sigma_{-}^{+}(T=0)=2 J+J_{1} \text {. }
$$


Let us now study the former type of interface by taking the usual mixed boundary conditions of $s=-1$ at the top of our system and $s=+1$ at the bottom (see Fig. 1). If we then take the limit $J_{1} \rightarrow \infty$, only configurations which satisfy $s(\mathbf{R}) \geq s(\mathbf{R}+a \hat{z})$ are allowed and the SOS constraint becomes exact. The interfacial free energy per unit area for this modified SOS (MSOS) model is then

$$
\sigma_{+}^{-}\left(J_{1} \rightarrow \infty\right) \equiv \sigma^{\mathrm{MSOS}}=2 J+f(J, T)
$$

where $f(J, T)$ is defined in Eq. (6.1). This free energy obviously vanishes again with "critical exponent" $\mu=1$, at a finite temperature $T_{c}$, but no critical behavior occurs at that point and the surface stiffness $\sigma+\sigma^{\prime \prime}$ remains finite. The bulk fluctuations that characterize normal critical behavior cannot occur in this model because they require portions of the forbidden type of interface. This simple model provides further illustration of why an interface Hamiltonian with a no overhang or SOS restriction cannot be used to model the behavior near the bulk critical point for any bulk dimension $d>1$.

It is also instructive to consider this modified Ising model for finite, positive $J_{1}$. In this case bulk excitations (bubbles) and overhangs in the interface are possible but cost an extra energy proportional to $J_{1}$. If the interfacial free energies are defined using the above-mentioned mixed boundary conditions, then a comparison of the actual microscopic configurations show that

$$
\sigma_{-}^{+}(T)=\sigma_{+}^{-}(T)+J_{1} .
$$

Regrouping the terms in Eq. (6.5), we see that our modified Ising model is precisely equivalent to a normal anisotropic Ising model with coupling $J_{1}=J+J_{1} / 4$ and surface fields of magnitude $J_{1} / 4$ favoring $s=-1$ in the top layer and $s=+1$ in the bottom layer (where these boundary layers are arbitrarily far apart). The bulk critical point, and consequent proliferation of overhangs and bubbles and vanishing of $\sigma^{\prime \prime}$ does not occur until the average interfacial free energy $\left(\sigma_{-}^{+}+\sigma_{+}^{-}\right)$vanishes, which means from (6.7) $\sigma_{+}^{-} \rightarrow-J_{1} / 2$. Thus even when the bubbles and overhangs are only slightly suppressed by a finite $J_{1}$ the vanishing of $\sigma_{-}^{+}$does not signal the bulk critical point.

\section{GENERALIZED INTERFACIAL MODEL}

In the preceding section the Ising model with an interface was reduced to an SOS model by suppressing configurations with excess segments of a particular horizontal interface. This is done by taking some nearest-neighbor coupling to infinity, which, however, has the unfortunate side effect of causing the bulk critical temperature to diverge. One may, on the other hand, suppress the configurations with overhangs and bubbles in a somewhat less intrusive way by attaching an additional energy, say $E_{r}$, to "reversals" in the interface, where the $z$ component of a unit vector normal to the interface changes sign. Such an Ising model with an interface again reduces to an SOS model in the limit $E_{r} \rightarrow \infty$, but now there exists a finite bulk critical temperature at which ferromagnetic longrange order disappears.

An explicit, exactly solvable Ising model that interpolates continuously between the usual nearest-neighbor

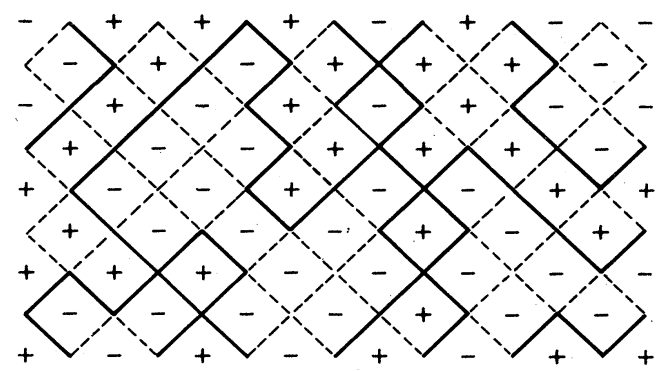

FIG. 2. Mapping of the Ising model onto an interfacial model on the dual lattice. Interfaces between + and - Ising spins correspond to solid lines in the vertex model.

model and a SOS-type model exists in $d=2$ dimensions. Consider an Ising model on a square lattice with, for convenience, the nearest-neighbor directions oriented at $45^{\circ}$ to the horizontal. Each spin configuration of this Ising model may be represented as a configuration of an interfacial model, where the segments of the interface are on the bonds of the dual lattice and each such segment separates antiparallel nearest-neighbor spins, as is illustrated in Fig. 2. This interfacial model is equivalent to an eight-vertex model $;{ }^{20}$ the eight allowed configurations of each node of the dual lattice and its four adjacent bonds are shown in Fig. 3. Let us consider the model with the following vertex energies

$$
\begin{aligned}
& e_{1}=-2 J, e_{2}=2 J+E_{c}, \\
& e_{3}=e_{4}=0, \\
& e_{5}=e_{6}=E_{b}, \\
& e_{7}=e_{8}=E_{r} .
\end{aligned}
$$

The usual Ising model with nearest-neighbor interaction $J$ is the case $E_{c}=E_{b}=E_{r}=0$. For general vertex energies we have an Ising model with additional next-nearestneighbor and four-spin couplings. ${ }^{20}$ An SOS model, with no overhangs or bubbles allowed is obtained in the limit $E_{r} \rightarrow \infty$.

This eight-vertex or Ising model (7.1) can be solved for vertex energies satisfying the free-fermion condition ${ }^{20}$

$$
e^{\beta E_{c}}+1=e^{-2 \beta E_{b}}+e^{-2 \beta E_{r}} .
$$

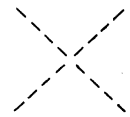

1

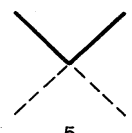

5

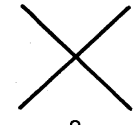

2

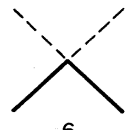

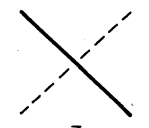

3

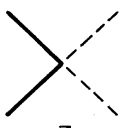

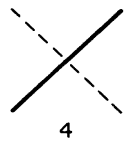

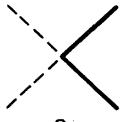

FIG. 3. Eight allowed interface configurations on each node of the dual lattice and their association with the eight-vertex configurations. The numbers refer to the standard vertex numbers of Lieb and Wu (Ref. 20). 
For concreteness, let us then restrict our attention to the case of vanishing crossing energy, $E_{c}=0$, and require that the bending energy $E_{b}$ is determined by the free-fermion condition (7.2). ${ }^{56}$ We will concentrate on the case where $E_{r}>0$, so that $E_{b}$, according to (7.2), is negative.

In the physically most relevant case where $J>\left|E_{b}\right|$, we see from Figs. 2 and 3 that the ground state of the spin system with +- boundary conditions consists of one area of + spins and one area of - spins (corresponding to vertex 1), separated by a single interface composed of vertices 5 and 6 [Fig. 4(a)]. The qualitative behavior of the model as a function of $E_{r}$ is further illustrated in Fig. 4. For all finite $E_{r}$, there are bubbles and overhangs in the system at temperatures $T>0$. The shape of these bubbles depends on $E_{b}, E_{r}$, and the temperature. For ex-
$0<E_{r}<\infty$

(a) $\mathrm{T}=0$

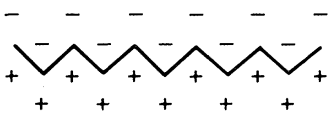

(b) $T>0, E_{r} \simeq\left|E_{b}\right|<k_{B} T$

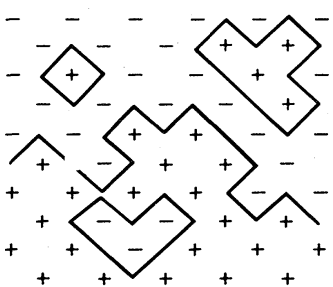

(c) $T>0, E_{r} \gg k_{B} T$

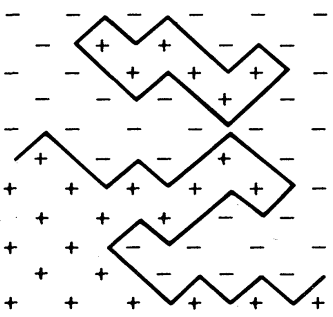

$E_{r}=\infty$

(d) $0<T<T_{c}$

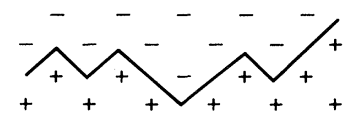

(e) $T \geqslant T_{c}$

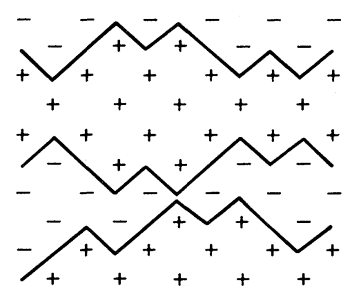

(f) $T \gg T_{c}$

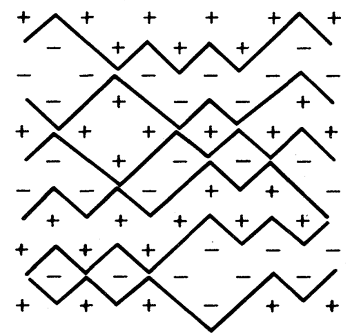

FIG. 4. Typical configurations of the lattice model with +boundary conditions and $\left|E_{b}\right|<J$ for various values of $E_{r}$. The ambiguity in drawing the lines at vertex 2 is also encountered in Fig. 1(a) where a bubbles intersects the long contour. Here we have drawn vertex 2 as two "bends" (like a combination of vertices 5 and 6). (a) The ground state for $0 \leq E_{r} \leq \infty$. (b) When $E_{r} \approx\left|E_{b}\right|<k_{B} T$ the bubbles are roughly symmetric and the behavior of the model is close to that of the nearestneighbor Ising model. (c) The bubbles and overhangs are elongated for $E_{r} \gg k_{B} T$. (d) For $E_{r}=\infty, T<T_{c}$ there is only one line separating + and - spins. (e) Just above $T_{c}$, there are multiple "interfaces," but they are still relatively dilute. Since $E_{r}=\infty$ the regions of + and - spins remain striped. (f) For $T \gg T_{c}$ there is a high density of interfaces. ample, when $E_{r} \approx\left|E_{b}\right|<k_{B} T$ bubbles are more-or-less symmetric since the number of "reversals" (vertices 7 and 8 ) is then roughly equal to the number of "bends" (vertices 5 and 6), see Fig. 4(b). For $E_{r} \gg k_{B} T$, however, the contours separating + and - spins are significantly elongated in the horizontal direction due to suppression of reversals [Fig. 4(c)]. When reversals are completely forbidden by taking $E_{r}=\infty$, the qualitative behavior is quite different [Figs. 4(e)-4(f)]. For sufficiently low temperatures (in fact for all $T<T_{c}$ ), there is only one contour, since creation of a new one is associated with an energy proportional to the system size because any interface must run across the entire system [Fig. 4(d)]. Only above a finite $T_{c}$ do multiple "interfaces" appear [Figs. 4(e)-4(f)]. However, even above $T_{c}$ the regions of + and - spins are always "striped," a feature not present when $E_{r}<\infty$. These multiple interfaces destroy long-range order, but the nature of the infinite $E_{r}$ phase transition is quite different from that of the Ising model, as we now proceed to show.

The critical temperature of our vertex model with the above restrictions is simply ${ }^{20}$

$$
\exp \left(2 J / k_{B} T_{c}\right)=1+\sqrt{2}
$$

for all reversal energies $E_{r}$. The phase diagram of this model as a function of $k_{B} T / 2 J$ and $\exp \left(-E_{r} / k_{B} T\right)$ is shown in Fig. 5. For any finite non-negative $E_{r}$, bubbles and overhangs are allowed and the order-disorder transition is a normal Ising transition with, for example, a logarithmically divergent specific heat and surface tension exponent $\mu=1$. In the limit $E_{r} \rightarrow \infty$, however, the bubbles and overhangs are suppressed completely. The model then reduces to a potassium dihydrogen phosphate (KDP-) like six-vertex model which has the same surface tension exponent $\mu=1$, but despite this the $E_{r}=\infty$ critical point is no longer in the Ising universality class because all inter-

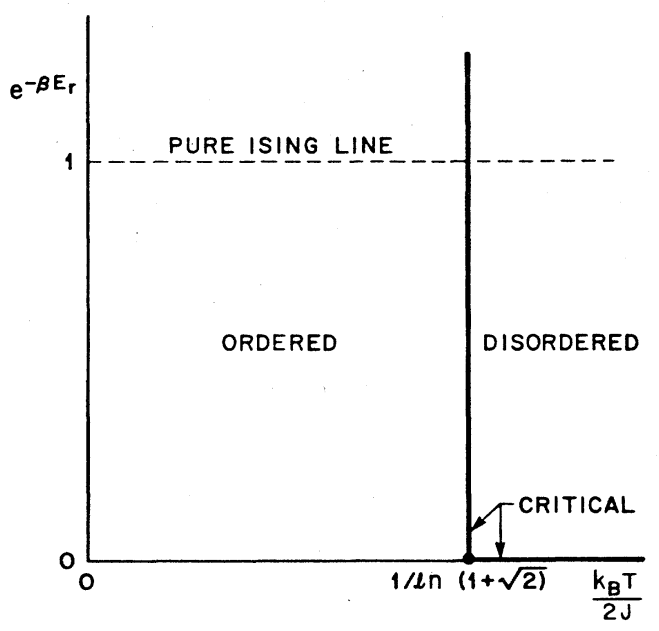

FIG. 5. Phase diagram of the of the eight-vertex model defined by (7.1) and (7.2) with $E_{c}=0$, as a function of $k_{B} T / 2 J$ and $\exp \left(-E_{r} / k_{B} T\right)$. Solid lines indicate critical lines. The phase transition at the vertical line where $\exp \left(2 J / k_{B} T\right)=1+\sqrt{2}$ is in the Ising universality class. For $E_{r}=\infty$, all interface reversals are suppressed and the transition is in the universality class of the KDP model. 
faces are forced to run from left to right throughout the whole system: $:^{57}$ e.g., the specific heat diverges with exponent $\alpha=\frac{1}{2}$ for $T \rightarrow T_{c}$ from above, characteristic of KDP-type models (as was argued by Haldane and Villain, ${ }^{58}$ this is the typical behavior of systems with such stringlike excitations). For all $T>T_{c}$, the $E_{r}=\infty$ system has algebraically decaying spatial correlations. For $T<T_{c}$ and $E_{r}=\infty$ there are no fluctuations about the ground state, but one can evaluate horizontal and vertical correlation lengths that have well-defined limits as $E_{r} \rightarrow \infty$. The horizontal correlation length diverges as $T \rightarrow T_{c}$ with critical exponent $\nu_{\|}=1$, just as at an Ising critical point, while the vertical correlation length diverges with exponent $v_{\perp}=\frac{1}{2}$ in this $E_{r} \rightarrow \infty$ limit. This again demonstrates the anisotropic scaling of interface Hamiltonians.

This simple example illustrates how the bubbles and overhangs in the interfaces are an essential part of the physics of the Ising universality class of criticality. When they are suppressed completely by taking $E_{r} \rightarrow \infty$, a different phase transition with different critical behavior results. Thus critical exponents obtained from a calculation that does not include bubbles and overhangs presumably represent a different universality class than the usual Ising critical point. This exactly solved model is restricted to $d=2$, but we expect similar models in any $d>1$ to show qualitatively the same behavior, namely a transition to a striped phase in the limit $E_{r} \rightarrow \infty$; this transition will exhibit anisotropic scaling and have $\mu=1$, and will therefore not be in the Ising universality class.

The result $\mu=1$ found in this model and in the SOS approximation (6.8), is in fact a general consequence in any dimension of the use of an interface-type Hamiltonian for which no bubbles and overhangs are permitted. This can be seen as follows ${ }^{59}$ (cf. also Secs. V and VI A). The surface tension $\sigma$ when computed from any simple interface Hamiltonian $H_{i}$ with short-range interactions ${ }^{60}$ and a cutoff is a decreasing function of temperature and goes through zero at some finite temperature $T^{*}$. Furthermore, the derivative

$$
\frac{d(\sigma / T)}{d T}=-\frac{1}{T^{2}}\left\langle H_{i}\right\rangle
$$

is just the internal energy, and is finite. Hence $\sigma$ must vanish linearly as $T \rightarrow T^{*}$ and consequently $\mu=1$. As discussed in Sec. V, this can be explicitly verified for the drumhead model in two bulk dimensions.

If $\mu=1$ in the drumhead Hamiltonian for all $d>1$, then why is it that Wallace and $\mathrm{Zia}^{11}$ did not find $\mu=1$ to all orders in $\epsilon=d-1$ ? We believe that the reason they did not obtain the result $\mu=1$ may be traced to the fact that they have not imposed a short-distance cutoff on the model. In fact, the nontrivial fixed point they find does not even exist in a model with a cutoff, unlike the case for the bulk Ising model where the universality class of the critical behavior is independent of the nature of the cutoff. If one renormalizes with a cutoff by a momentum shell integration, the square-root form of the Hamiltonian is not preserved and it appears that the only finitetemperature fixed-point Hamiltonian of such a renormalization group is the simple capillary-wave Hamiltonian
(3.3), which satisfies anisotropic scaling and has $\mu=1$. In the analysis of Ref. 11 , the interface height $h(\mathbf{r})$ was scaled as a length, but from Eq. (4.6) it is clear that the lengths must scale anisotropically in treating the drumhead Hamiltonian with a cutoff, i.e., if lengths parallel to the interface rescale with a factor $l$ then the length $h(\mathbf{r})$ perpendicular to it should scale as $l^{(3-d) / 2}$. This is another indication that such interface Hamiltonians should not be used to describe bulk critical properties, for which isotropic scaling holds (cf. Sec. V).

The relevance of overhangs and bubbles in two dimensions can be argued more generally by considering models with the reversal fugacity, $y \equiv \exp \left(-E_{r} / k_{B} T\right)$ as a parameter. An expansion of the partition function or the surface tension perturbatively in $y$ then reveals that the $y=0$ transition is actually multicritical, as in Fig. 5, with $y$ representing a relevant variable. We have verified this by a calculation along the lines of the one of Huse and Fish$\mathrm{er}^{61}$ for $2 \times 1$ commensurate overlayers (in their case the interfacial reversals represent dislocations in the overlayer). For a calculation of the surface tension one may actually assign different fugacities to reversals in overhangs and reversals in bubbles; both are relevant, with the same scaling exponent. This allows the contributions to the surface tension from overhangs and bubbles to cancel precisely for certain orientations of the interface in the nearest-neighbor Ising model. However, the fact that they cancel does not make them irrelevant in a renormalization-group picture. Rather, their cancellation should be viewed as a coincidence, which is permitted by the fact that $\mu=1$ for the $d=2$ Ising model.

Many two-dimensional models can be represented in terms of stringlike excitations with appropriate Boltzmann weight assigned to reversals and intersections of these strings. Thus if we alter the above Ising model to allow reversals and overhangs of the interface, but no separate bubbles (loops), the system becomes a selfavoiding walk and is again in another universality class. ${ }^{18}$ Similarly, one can construct Potts models, etc. by allowing different types of interfacial segments and intersections. These systems have quite a variety of critical behavior, but all reduce to simple SOS-type models in the limit where reversal and intersection fugacities are taken to zero.

\section{FINAL REMARKS}

Our examination of the derivation and use of interface Hamiltonians has pointed out their limitations for analyzing bulk critical properties for which a full $d$-dimensional description incorporating bubbles and overhangs is required. On the other hand, an interface Hamiltonian gives an accurate description of long-wavelength interface fluctuations, and provides a simple means to calculate the effects of such fluctuations. Nontrivial consequences of these fluctuations for the liquid-vapor system are found in the behavior of the interface width and in the existence of long-ranged density correlations parallel to the interface. 9,27 In other surface phenomena not associated with a bulk phase transition, such as roughening, ${ }^{1}$ layering, ${ }^{2,3}$ and critical wetting 4,5 transitions, the long-wavelength fluctuations play a significant role and here again the 
analysis of an interface Hamiltonian is an important step in developing a tractable theory.

\section{ACKNOWLEDGMENTS}

We are grateful to P. C. Hohenberg, D. S. Fisher, M. E. Fisher, and R. K. P. Zia for helpful comments and spirited discussions. One of us (D.A.H.) thanks the Aspen Center for Physics, where part of this work was done.

\section{APPENDIX: ANALYSIS OF THE DERIVATION OF $H_{\mathrm{dh}}$ FROM $H_{\mathrm{LGW}}$}

In this appendix we briefly discuss the derivations of the drumhead Hamiltonian $H_{\mathrm{dh}}$ from the LandauGinzburg-Wilson Hamiltonian (3.1) in the light of the interface picture described in Secs. II-IV, and show that the validity of $H_{\mathrm{dh}}$ is confined to length scales large compared to $\xi_{B}$. The derivations in the literature ${ }^{62-64}$ are essentially based on a low-temperature (mean-field) picture of the interface. We first discuss this picture, emphasizing the assumption of single valuedness of the interface, and then comment on the derivation close to $T_{c}$.

At low temperatures $\left(T<<T_{c}\right)$, a mean-field analysis of $H_{\text {LGW }}$ becomes adequate and $H_{\mathrm{dh}}$ can be obtained from what is essentially a mean-field-type analysis of a slightly curved interface. Let $M(z)$ be the mean-field local order parameter profile corresponding to $H_{\mathrm{LGW}}$ [Eq. (3.1)] below $T_{c}(\tau<0)$ when the interface is flat and perpendicular to the $z$ direction,

$$
M(z)=(|\tau| / u)^{1 / 2} \tanh \left[(|\tau| / 2)^{1 / 2} z\right],
$$

which is the solution of

$$
\left(\frac{d^{2}}{d z^{2}}+|\tau|-u M^{2}(z)\right) M(z)=0 .
$$

At low temperatures, $|\tau|^{-1 / 2} \simeq \xi_{B}$.

Equation (A1) gives the interface profile in the meanfield configuration $\phi(\mathbf{R})=M(z)$ whose energy minimizes $H_{\mathrm{LGW}}$ under the appropriate boundary conditions. To examine the effects of long-wavelength fluctuations, we follow Diehl et al. ${ }^{63}$ and Kawasaki and Ohta ${ }^{62}$ and implicitly define an interface position $h(\mathbf{r})$ for each spin configuration $\phi(\mathbf{R})$ by the requirement that

$$
\int_{-\infty}^{+\infty} d z \phi(\mathbf{R}) M^{\prime}(z-h(\mathbf{r}))=0 .
$$

Here $M^{\prime} \equiv d M / d z$ and we have used a coordinate system $\mathbf{R}=(\mathbf{r}, z)$. An interface Hamiltonian $H_{i}(\{h(\mathbf{r})\})$ incorporating some fluctuations about the flat mean-field profile is thus obtained by integrating over those bulk configurations which are consistent with a given $h(\mathbf{r})$.

We emphasize that any definition of the form (A3) already implies a coarse graining in the following sense. For a given field configuration $\phi(\mathbf{R})$, the solution $h(\mathbf{r})$ of (A3) can, in principle, be multiple valued; this corresponds to the presence of interfaces with overhangs or the existence of bubbles [closed interface loops, like the dashed lines in Fig. 1(b)]. However, since $M^{\prime}(z)$ has at low temperatures a width of the order of $|\tau|^{-1 / 2} \simeq \xi_{B}$, only overhangs and bubbles of size larger than $\xi_{B}$ will according to (A3) give rise to multiple-valued solutions of $h(\mathbf{r})$, while the overhangs and bubbles of size smaller than $\xi_{B}$ merely shift the interface position slightly.

Note also that the definition (A3) also breaks the rotational symmetry of the system: A cigar-shaped bubble of width smaller than $\xi_{B}$ but length much larger than $\xi_{B}$ does give rise to a multiple-valued interface if it points in the $z$ direction, but not if it is directed along the interface. As discussed in Sec. V, this lack of rotational symmetry is also present in the set of interface configurations used to calculate the free energy from $H_{\mathrm{dh}}$.

In practice even those configurations which do give rise to multiple-valued $h(\mathbf{r})$ from (A3) are ignored and a single-valued $h(\mathbf{r})$ is assumed to give an adequate description. This amounts to a mean-field analysis of a gently curved interface and is consistent only at low temperatures. This is explicitly assumed in the formal derivation of $H_{\mathrm{dh}}$ by Diehl et al., ${ }^{63}$ and shown below, can also be seen from the simpler (but essentially equivalent) derivation of Kawasaki and Ohta. ${ }^{62}$ Kawasaki and Ohta ${ }^{62}$ determine the solution $\phi(\mathbf{R})=\chi(z-h(\mathbf{r}), \mathbf{r})$ which minimizes $H_{\mathrm{LGW}}$ under the constraint (A3). The variational problem for $\chi$ leads to the equation

$$
\begin{aligned}
{\left[1+\left(\partial_{\mathrm{r}} h\right)^{2}\right] \partial_{z}^{2} \chi+} & \partial_{\mathrm{r}}^{2} \chi+|\tau| \chi-u \chi^{3} \\
& -2 \partial_{\mathrm{r}} h \cdot \partial_{\mathrm{r}} \partial_{z} \chi-\partial_{\mathrm{r}}^{2} h \partial_{z} \chi=v M^{\prime}(z),
\end{aligned}
$$

where $v$ is the Lagrange multiplier associated with (A3). From here, one arrives at $H_{\mathrm{dh}}$ by neglecting the terms

$$
\partial_{\mathrm{r}}^{2} \chi, \quad \partial_{\mathrm{r}} h \cdot \partial_{\mathrm{r}} \partial_{z} \chi, \quad \partial_{\mathrm{r}}^{2} h \partial_{z} \chi,
$$

so that (A4) reduces to

$$
\left\{\left[1+\left(\partial_{\mathrm{r}} h\right)^{2}\right] \partial_{z}^{2}+|\tau|-u \chi^{2}\right\} \chi=v M^{\prime}(z) .
$$

Kawasaki and Ohta ${ }^{62}$ argue that $v$ should be zero since $\chi$ will be an odd function of $z$. The solution of (A6) then reads [cf. Eqs. (A2) and (A3)]

$$
\chi=M\left(\left[1+\left(\partial_{\mathbf{r}} h\right)^{2}\right]^{-1 / 2} z\right) .
$$

Upon substitution of this solution for $\phi$ into $H_{\mathrm{LGW}}$ and carrying out the $z$ integration, one then arrives at $H_{\mathrm{dh}}$ with $\sigma_{\mathrm{MF}}$ given by the usual mean-field (MF) expression

$$
\sigma_{\mathrm{MF}}=\int_{-\infty}^{+\infty} d z\left[M^{\prime}(z)\right]^{2} .
$$

Obviously, the validity of the above mean-field analysis is limited to length scales such that the terms (A5) are small compared to those retained in Eq. (A6). Thus we need to have, for instance,

$$
\partial_{\mathbf{r}}^{2} \chi \ll \partial_{z}^{2} \chi
$$

Since $\chi$ varies over scales of the order of $\xi_{B}\left(\simeq \tau^{-1 / 2}\right)$ in the $z$ direction, the length scales $l$ along the interface over which (A9) is obeyed are

$$
l \gg \xi_{B},
$$

in agreement with the arguments given in Secs. II-IV.

While the above analysis again confirms that $H_{\mathrm{dh}}$ is a long-wavelength Hamiltonian away from criticality, the mean-field derivation obviously breaks down close to criticality. However, as argued in Secs. II-IV, the drumhead Hamiltonian remains valid even close to criticality 
on sufficiently long length scales obeying (A10). This can be understood within the self-consistent renormalizationgroup picture discussed in Sec. III. By integrating out the fluctuations of wavelength up to order $\xi_{B}$ in $H_{\mathrm{LGW}}$ and rescaling, one is driven away from criticality. In doing so, the singular part of the free energy is determined and overhangs and bubbles gradually disappear. When one ar- rives at length scales $l^{\prime}$ obeying (A10), $\tau\left(l^{\prime}\right)$ in $H_{\mathrm{LGW}}$ is large (low temperatures) and a mean-field analysis like the one sketched above is appropriate, provided we set $\tau^{-1 / 2}\left(l^{\prime}\right)$ equal to the true correlation length $\xi^{\prime}$ at the length scale, rather than the mean-field correlation length. Similarly, the macroscopic $\sigma$ rather than the mean-field $\sigma_{\mathrm{MF}}$ as in (A8) then appears in $H_{\mathrm{dh}}$.
${ }^{1}$ For a review of the roughening transition, see J. D. Weeks, in Ordering in Strongly Fluctuating Condensed Matter Systems, edited by T. Riste (Plenum, New York, 1980), p. 293.

2J. D. Weeks, Phys. Rev. B 26, 3998 (1982).

${ }^{3}$ D. A. Huse, Phys. Rev. B 30, 1371 (1984).

${ }^{4}$ R. Lipowsky, D. M. Kroll, and R. K. P. Zia, Phys. Rev. B 27, 4499 (1983).

${ }^{5}$ E. Brézin, B. I. Halperin, and S. Leibler, Phys. Rev. Lett. 50, 1387 (1983).

${ }^{6} \mathrm{~K}$. Binder, in Phase Transitions and Critical Phenomena, edited by C. Domb and J. L. Lebowitz (Academic, New York, 1983), Vol. 8.

${ }^{7}$ D. Jasnow, Rep. Progr. Phys. 47, 1059 (1984).

${ }^{8}$ R. K. P. Zia, in Proceedings of the Scottish Universities Summer School in Physics, 1983, edited by K. Bowler and A. McKane (unpublished).

${ }^{9}$ J. D. Weeks, J. Chem. Phys. 67, 3106 (1977).

${ }^{10}$ F. P. Buff, R. A. Lovett, and F. H. Stillinger, Phys. Rev. Lett. 15, 621 (1965).

${ }^{11}$ D. J. Wallace and R. K. P. Zia, Phys. Rev. Lett. 43, 808 (1979).

12D. J. Wallace, in Phase Transitions, Cargèse 1980, edited by M. Lévy, J. C. Le Guillou, and J. Zinn-Justin (Plenum, New York, 1982).

${ }^{13}$ D. J. Wallace, in Recent Advances in Field Theory and Statistical Mechanics, edited by J. B. Zuber and R. Stora (NorthHolland, Amsterdam, 1984), Vol. 39.

${ }^{14}$ D. Forster and A. Gabriunas, Phys. Rev. A 23, 2627 (1981); 24, 598 (1981).

${ }^{15}$ A. D. Bruce and D. J. Wallace, Phys. Rev. Lett. 47, 1743 (1981); J. Phys. A 16, 1721 (1983); see also B. Schmittmann, ibid. 17, 403 (1984).

16B. Widom, J. Chem. Phys. 43, 3892 (1965).

${ }^{17} \mathrm{~B}$. Widom, in Phase Transitions and Critical Phenomena, edited by C. Domb and M. S. Green (Academic, New York, 1972), Vol. 2.

${ }^{18} \mathrm{~S}$. Teitel and D. Mukamel (unpublished).

${ }^{19}$ E. Müller-Hartmann and J. Zittartz, Z. Phys. B 27, 261 (1977).

${ }^{20}$ See E. H. Lieb and F. Y. Wu, in Phase Transitions and Critical Phenomena, edited by C. Domb and M. S. Green (Academic, London, 1972), Vol. 1, p. 332.

${ }^{21}$ T. Ohta and K. Kawasaki, Prog. Theor. Phys. 58, 467 (1977).

22J. Rudnick and D. Jasnow, Phys. Rev. B 17, 1351 (1978).

${ }^{23}$ K. G. Wilson, Rev. Mod. Phys. 47, 773 (1975).

${ }^{24}$ For an introduction, see S. K. Ma, Modern Theory of Critical Phenomena (Benjamin, London, 1976).

${ }^{25}$ M. E. Fisher and A. E. Ferdinand, Phys. Rev. Lett. 19, 169 (1967).

26M. E. Fisher, J. Phys. Soc. Jpn. Suppl. 26, 87 (1969).

27J. D. Weeks, Phys. Rev. Lett. 52, 2160 (1984).

${ }^{28}$ Alternative definitions of the local interface width, corresponding to Widom's intrinsic width, are given by F. H. Stil- linger, J. Chem. Phys. 76, 1087 (1982); Int. J. Quantum Chem. 16, 137 (1982); and by D. B. Abraham, Phys. Rev. B 29, 525 (1984).

${ }^{29} \mathrm{~A}$ similar argument has arisen in the discussion about the lower critical dimension $d_{c}$ of random-field Ising models. As discussed in detail by G. Grinstein and S. K. Ma [Phys. Rev. B 28, 2588 (1983)], the vanishing of $f$ for large $L$ for $d>2$ in that problem implies that the interface between domains of "up" and "down" spins in random-field models is effectively smooth. This favors the conclusion that $d_{c}$ is equal to 2 .

30J. D. Weeks, D. Bedeaux, and B. J. A. Zielinska, J. Chem. Phys. 80, 3790 (1984).

${ }^{31}$ D. Jasnow and J. Rudnick, Phys. Rev. Lett. 41, 698 (1978).

${ }^{32}$ E. S. Wu and W. W. Webb, Phys. Rev. A 8, 2065 (1973).

${ }^{33} \mathrm{See}$, on this point in particular, M. B. Schneider and W. W. Webb, Bull. Am. Phys. Soc. 29, 486 (1984), who reanalyzed the experiments of Ref. 32 .

${ }^{34}$ M. E. Fisher, Physics 3, 255 (1967).

${ }^{35}$ The appearance of such singularities in quantities reflecting the droplet density can be understood intuitively in terms of the finiteness of the energy $E$ associated with a droplet boundary, even at $d=1$ where $E=2 J$ ( $J$ being the nearestneighbor interaction strength). The associated Boltzmann factors $\exp \left(-E / k_{B} T_{c}\right)$ at criticality therefore becomes of the form $\exp (-c / \epsilon)$ for $d$ near 1 where $T_{c}=O(\epsilon)$

${ }^{36}$ The possibility that the fixed point studied by Wallace and Zia is different from the Ising fixed point has also been realized by R. K. P. Zia (private communication). See, in this connection also, F. David, Phys. Lett. 102B, 193 (1981).

${ }^{37}$ See J. L. Cardy and H. W. Hamber, Phys. Rev. Lett. 45, 499 (1980), and references therein.

${ }^{38} \mathrm{Cf}$. also the analysis by Teitel and Mukamel, Ref. 18. These authors introduce a field conjugate to tilting of the interface in the discretized $2 d$ drumhead Hamiltonian. They show that the susceptibility to tilting never diverges at any finite temperature, implying that the free energy associated with $H_{\mathrm{dh}}$ never becomes rotationally invariant at finite $T$.

${ }^{39}$ See also D. Jasnow, B. B. Pant, and T. Ohta, Phys. Rev. A 26, 3532 (1982).

${ }^{40}$ M. P. A. Fisher, D. S. Fisher, and J. D. Weeks, Phys. Rev. Lett. 48, 369 (1982).

${ }^{41}$ D. B. Abraham, Phys. Rev. Lett. 47, 545 (1981).

${ }^{42}$ C. Rottman and M. Wortis, Phys. Rev. B 24, 6274 (1981).

${ }_{43}$ J. E. Avron, H. van Beijeren, L. S. Schulman, and R. K. P. Zia, J. Phys. A 15, L81 (1982).

${ }^{44}$ The fact that this approximation does not yield exact results for the antiferromagnetic Ising model was demonstrated by R. J. Baxter, I. G. Enting, and S. K. Tsang, J. Stat. Phys. 22, 465 (1980); Z. Racz, Phys. Rev. B 21, 4012 (1980); and J. Zittartz, Z. Phys. B 40, 233 (1981).

${ }^{45}$ T. W. Burkhardt, Z. Phys. B 29, 129 (1978).

${ }^{46}$ B. W. Southern, Z. Phys. B 30, 61 (1978).

${ }^{47}$ R. M. Hornreich, R. Liebmann, H. G. Schuster, and W. Selke, 
Z. Phys. B 35, 91 (1979).

${ }^{48}$ K. Y. Lin and F. Y. Wu, Z. Phys. B 33, 181 (1979).

49J. Zittartz, Z. Phys. B 41, 65 (1981).

50J. Dóczi-Réger and P. C. Hemmer, Physica 108A, 531 (1981).

${ }^{51}$ J. Kroemer and W. Pesch, J. Phys. A 15, L25 (1982).

${ }^{52}$ T. Temesvári, J. Phys. A 15, L625 (1982).

${ }^{53}$ P. A. Slotte, J. Phys. A 15, L507 (1982).

${ }^{54}$ W. Selke and W. Pesch, Z. Phys. B 47, 335 (1982).

${ }^{55}$ See, e.g., Ref. 54 . The authors of this paper also realized that $\mu=1$ in this approximation.

${ }^{56}$ This choice implies, as (7.2) shows, that $\exp \left(-E_{r} / k_{B} T\right)$ must be less than 2 , but this constraint does not effect our argument since we assume $E_{r} \geq 0$.

${ }^{57}$ H. W. J. Blöte and H. J. Hilhorst [J. Phys. A 15, L631 (1982)] have formulated a roughening model in this same universality class which can also be mapped on a KDP-type six-vertex model and whose excitations are long domain walls.

${ }^{58}$ F. D. M. Haldane and J. Villain, J. Phys. (Paris) 42, 1673 (1981).

${ }^{59}$ Jasnow et al. (Ref. 39) have employed a duality type transformation to the drumhead Hamiltonian. In terms of the dual variables the absence of a phase transition is clear from the fact that the resulting potential for these dual variables is a smooth function with only one minimum in any dimension and for all temperatures.

${ }^{60}$ In $d=2$, one needs logarithmically divergent interactions to get isotropic behavior at some temperature $T^{*}$. See Ref. 18.

${ }^{61}$ D. A. Huse and M. E. Fisher, Phys. Rev. B 29, 239 (1984).

${ }^{62}$ K. Kawasaki and T. Ohta, Prog. Theor. Phys. 67, 147 (1982).

${ }^{63}$ H. W. Diehl, D. M. Kroll, and H. Wagner, Z. Phys. B 36, 329 (1980).

${ }^{64}$ D. J. Wallace, Ref. 12. 